Methods: This retrospective study included 74 SLE patients and 79 age- and sex-matched healthy subjects. According to the SLEDAI-2K score, SLE patients were divided into Group 1 with a score $\leq 9$ (patients with mild disease activity, $N=41$ ) and Group 2 with a score $>9$ (patients with moderate to severe disease activity, $N=33$ ). Neutrophil, lymphocyte, monocyte, platelet, albumin, fibrinogen NLR, PLR, monocyte to lymphocyte ratio (MLR), FAR, C-reactive protein (CRP), erythrocyte sedimentation rate (ESR), complement 3 (C3), complement 4 (C4) and SLEDAI-2K were collected. Receiver operation characteristic (ROC) curves were conducted to discriminate SLE patients from healthy controls and SLE patients with different disease activity. Correlations between the inflammatory markers and SLEDAI-2K were analyzed.

Results: FAR, NLR, PLR, MLR and fibrinogen in SLE patients were higher compared to those of the healthy controls $(P<0.05)$, while albumin was lower $(P<0.05)$. Patients in Group 2 had higher levels of FAR, NLR, PLR, fibrinogen and CRP than those in Group $1(P<0.05)$ except MLR $(P=0.579)$ and ESR $(P=0.130)$, albumin and C3 were lower $(P<0.05)$. Furthermore, FAR were higher in SLE patients with nephritis than those without nephritis $(P=0.009)$. The ROC curve for differentiating SLE patients from healthy individuals showed that the area under curve (AUC) of FAR $(0.801,95 \% \mathrm{Cl}$ : $0.728-0.874)$ and albumin $(0.833,95 \% \mathrm{Cl}: 0.764-0.903)$ were higher than NLR $(0.798,95 \% \mathrm{Cl}: 0.725-0.871)$, PLR $(0.680,95 \% \mathrm{Cl}: 0.588-0.771)$, MLR $(0.785,95 \% \mathrm{Cl}: 0.712-0.859)$ and fibrinogen $(0.645,95 \% \mathrm{Cl}: 0.556-0.733)$. Besides, the ROC curve for predicting the disease activity of SLE patients indicated that the AUC of FAR $(0.911,95 \% \mathrm{Cl}$ : $0.842-0.980)$ was higher than NLR $(0.697,95 \% \mathrm{Cl}: 0.574-0.821)$, PLR $(0.695,95 \% \mathrm{Cl}: 0.571-0.820)$, $\operatorname{MLR}(0.553,95 \% \mathrm{Cl}: 0.415-0.691)$, fibrinogen $(0.718,95 \% \mathrm{Cl}: 0.590-0.847)$, CRP $(0.672,95 \% \mathrm{Cl}: 0.540-0.803)$, ESR $(0.640,95 \% \mathrm{Cl}: 0.510-0.770)$, C3 (0.644, 95\%Cl: $0.515-0.773), \mathrm{C} 4(0.544,95 \% \mathrm{Cl}: 0.407-0.681)$ and albumin (0.894, 95\% Cl: $0.819-0.969)$. FAR was positively correlated with SLEDAI-2K $(r=0.682, P<0.001)$, yielding a highest relevance than other inflammatory parameters.

Conclusion: FAR was significantly elevated in SLE patients compared with healthy subjects and related with the disease activity of SLE. FAR might be a useful inflammatory index to evaluate disease activity in patients with SLE.

REFERENCES:

[1] Liu M, Huang Y, Huang Z, Zhong Z, Deng W, Huang Z, et al. The role of fibrinogen to albumin ratio in ankylosing spondylitis: Correlation with disease activity. Clin Chim Acta. 2020 Jun; 505:136-140.

Disclosure of Interests: None declared

DOI: 10.1136/annrheumdis-2021-eular.1406

\section{POS1444 ADOPTION OF DIGITAL MANIKINS TO SELF-REPORT PAIN: A SYSTEMATIC REVIEW}

S. Mustafa Ali ${ }^{1}$, R. Lee ${ }^{1}$, A. Chiarotto ${ }^{2}$, J. Mcbeth ${ }^{1}$, S. Van der Veer ${ }^{3}$, W. Dixon ${ }^{1}$. ${ }^{1}$ University of Manchester, Centre for Epidemiology Versus Arthritis, Manchester, United Kingdom; ${ }^{2}$ Erasmus University Medical Centre, Department of General Practice, Rotterdam, Netherlands; ${ }^{3}$ University of Manchester, Centre for Health Informatics, Manchester, United Kingdom

Background: Chronic pain is common in rheumatic and musculoskeletal conditions, and a major driver of disability worldwide. Knowledge gaps exist with respect to correct estimates of chronic pain [1], what causes it and how best to manage it [2]. To address this, researchers need validated methods to measure pain in large, representative populations. Though many authors have recognised the potential benefits of paper-based and digital pain manikins [3]-[5], it is unknown to what extent studies have adopted digital manikins as a data collection tool.

Objectives: The objective of our review was to identify and characterise published studies that have used digital pain manikins as a data collection tool. Methods: We systematically searched six electronic databases, including Medline, CINAHL, Embase, Scopus, IEEE Xplore digital library, ACM Digital Library, on 3-4 of November 2020 by using a pre-defined search strategy. We included a study in our review if it used a digital manikin for self-reporting any pain aspect (e.g., intensity, type) by people suffering from pain, and if its full text was published in English. We conducted this review by following the PRISMA reporting guidelines and conducted a descriptive synthesis of findings, including manikin-derived outcome measures.

Results: Our search yielded 4,685 unique studies. After full text screening of 705 articles, we included 14 studies in our review. Most articles were excluded because they used either paper-based manikins or didn't include enough details to determine that the manikin was digital $(n=386)$. The majority of included studies were published in Europe $(n=11)$. Most studies collected data on a manikin once $(n=11)$; from people with pain conditions $(n=9)$; and in clinical settings $(n=9)$. There was only one study that collected digital pain manikin data in a large sized (i.e., 20,000) population-based survey.
In most studies participants shaded any painful area on manikin $(n=9)$ and did not enable participants to record location-specific pain aspects $(n=11)$. None of the manikins enabled participants to record location-specific pain intensity. Pain distribution (i.e. number or percentage of pre-defined body areas or locations experiencing pain) and pain extent (i.e. number or percentage of shaded pixels) were commonly used manikin-derived outcome measures. In six studies, a heat map was used to summarise the extent of pain across the population.

Conclusion: Digital pain manikins have been available since the 1990s but their adoption in research has been slow. Few manikins enabled location-specific pain recording suggesting that the digital nature of the manikin is not yet fully utilised. Future development of a validated digital pain manikin supporting self-reporting of the location and intensity of pain, usable across any device and screen size, may increase uptake and value.

\section{REFERENCES:}

[1] S. E. E. Mills, K. P. Nicolson, and B. H. Smith, "Chronic pain: a review of its epidemiology and associated factors in population-based studies," Br. J. Anaesth., vol. 123, no. 2, pp. e273-e283, Aug. 2019.

[2] D. B. Reuben et al., "National Institutes of Health Pathways to Prevention Workshop: The Role of Opioids in the Treatment of Chronic Pain," Ann. Intern. Med., vol. 162, no. 4, p. 295, Feb. 2015

[3] R. Waller, P. Manuel, and L. Williamson, "The Swindon Foot and Ankle Questionnaire: Is a Picture Worth a Thousand Words?," ISRN Rheumatol., vol. 2012, pp. 1-8, 2012.

[4] M. Barbero et al., "Clinical Significance and Diagnostic Value of Pain Extent Extracted from Pain Drawings: A Scoping Review," Diagnostics, vol. 10, no. 8, p. 604, Aug. 2020.

[5] S. M. Ali, W. J. Lau, J. McBeth, W. G. Dixon, and S. N. van der Veer, "Digital manikins to self-report pain on a smartphone: A systematic review of mobile apps," Eur. J. Pain, vol. 25, no. 2, pp. 327-338, Feb. 2021.

Disclosure of Interests: None declared

DOI: 10.1136/annrheumdis-2021-eular.2679

\section{POS1445 RETINOL BINDING PROTEIN 4 AS AN ACUTE PHASE REACTANT AND BIOMARKER IN PATIENTS WITH FAMILIAL MEDITERRANEAN FEVER AND AMYLOIDOSIS COMPARED TO INFECTIONS}

N. Koca ${ }^{1}$, R. Deniz ${ }^{1}$, M. Erdugan ${ }^{1}$, Y. Yalçınkaya ${ }^{1}$, B. Artim-Esen ${ }^{1}$, M. L. Ocal ${ }^{1}$, M. Inanc ${ }^{1}$, A. Gül ${ }^{1} .{ }^{1}$ Istanbul Faculty of Medicine, Istanbul University, Division of Rheumatology, Department of Internal Medicine, Istanbul, Turkey

Background: Retinol binding protein 4 (RBP4) is a plasma retinol transporter that transports retinol from liver to periphery. RBP4 has been studied as a biomarker in metabolic and neoplastic conditions, however its association with inflammation is not clear. Serum amyloid A (SAA), another retinol binding protein, has been known as a sensitive biomarker of inflammation in familia Mediterranean fever (FMF) and other autoinflammatory disorders. C-reactive protein (CRP), erythrocyte sedimentation rate (ESR) and SAA are commonly used as acute phase reactants, but they are not successful in differentiating non-infectious inflammatory conditions from infections.

Objectives: We aimed to evaluate the potential of serum RBP4 as a biomarker of acute phase response and to determine its performance in differentiation of inflammation of patients with FMF and AA amyloidosis from infections.

Methods: A total of 169 participants in 5 groups, consisting of FMF ( $n=60$ ), FMF with $A A$ amyloidosis $(n=58)$, non-FMF AA amyloidosis $(n=23)$, infections ( $n=10,3$ pneumonia, 3 sepsis, 1 pyelonephritis, 1 fungal infection, 1 cellulitis, 1 disseminated zoster), and healthy controls $(H C)(n=18)$, were included and evaluated cross sectionally. Hemogram and serum CRP, ESR, SAA, ferritin, creatinine, AST, ALT, albumin levels were recorded from the patient charts. FMF and FMF + amyloidosis patients were evaluated during attack-free period. Serum RBP4 levels were investigated by ELISA (Elabscience, USA). Mean values and relative changes compared to healthy controls were evaluated for SAA, CRP RBP4 levels in all groups.

Results: Serum RBP4 level was found to be higher in FMF group compared to the patients with infection $(p=0.002)$ and $\mathrm{HC}(p<0.001)$ as well as in patients with amyloidosis. Compared to $\mathrm{HC}, 47 \%, 28 \%$ and $27 \%$ increase was observed in mean RBP4 levels in FMF, FMF + amyloidosis and nonFMF amyloidosis patients, despite no significant change in patients with infections. However, CRP and SAA elevations were much more prominent in patients with infections (58 and 134 times, respectively) compared to the patients with FMF (13 and 35 times, respectively), FMF + amyloidosis and non-FMF amyloidosis (Table 1). There was no significant difference in RBP4 levels between FMF, FMF-amyloidosis and non-FMF amyloidosis groups. CRP, ESR, ferritin and SAA levels were higher in the infection group compared to $\mathrm{HCs}$. 
Table 1. Demographic features and laboratory findings of the participants

\begin{tabular}{|c|c|c|c|c|c|}
\hline Variables & $\begin{array}{l}\text { FMF } \\
(n=60)\end{array}$ & $\begin{array}{c}\text { FMF- } \\
\text { Amyloidosis } \\
(n=58)\end{array}$ & $\begin{array}{l}\text { Non-FMF-AA } \\
\text { Amyloidosis } \\
\quad(n=23)\end{array}$ & $\begin{array}{c}\text { Infection } \\
(n=10)\end{array}$ & $\begin{array}{l}\text { Healthy } \\
\text { control } \\
(n=18)\end{array}$ \\
\hline Female/Male & $46 / 14$ & $33 / 25$ & $8 / 15$ & $3 / 7$ & $8 / 10$ \\
\hline \multirow[t]{2}{*}{ Age (SD)* } & $38 \pm 13$ & $43 \pm 11$ & $53 \pm 13$ & $65 \pm 15$ & $33 \pm 9$ \\
\hline & $(18-74)$ & $(21-69)$ & & & \\
\hline $\begin{array}{l}\text { Creatinine (mg/ } \\
\text { dL) }\end{array}$ & $0,8 \pm 0,2$ & $1,7 \pm 1,7$ & $2,0 \pm 1,6$ & $1,7 \pm 1,0$ & $0,7 \pm 0,2$ \\
\hline Albumin $(\mathrm{mg} / \mathrm{dL})^{\star}$ & $4,7 \pm 0,4$ & $4,3 \pm 0,6$ & $3,3 \pm 0,9$ & $3,0 \pm 0,9$ & $4,8 \pm 0,2$ \\
\hline Ferritin $(\mathrm{ng} / \mathrm{mL})^{\star}$ & $70 \pm 94$ & $245 \pm 315$ & $139 \pm 168$ & $554 \pm 38$ & $83 \pm 72$ \\
\hline RBP4 (ng/mL)* & $772 \pm 183$ & $671 \pm 214$ & $666 \pm 256$ & $512 \pm 204$ & $524 \pm 117$ \\
\hline \multirow[t]{2}{*}{ RBP4 (median) } & 770 & 653 & 645 & 487 & 498 \\
\hline & $(434-1142)$ & $(227-1259)$ & $(331-1214)$ & $(226-876)$ & $(566-738)$ \\
\hline CRP (mg/L)* & $16 \pm 47,1$ & $12,8 \pm 32,8$ & $25,7 \pm 36,4$ & $69 \pm 36,8$ & $1,2 \pm 1,2$ \\
\hline SAA $(\mathrm{mg} / \mathrm{dL})^{\star}$ & $10,3 \pm 31,4$ & $5,0 \pm 13,9$ & $7,1 \pm 14,1$ & $40,2 \pm 18,5$ & $0,3 \pm 0,1$ \\
\hline $\mathrm{ESR}^{\star}$ & $15 \pm 13$ & $19 \pm 16$ & $41 \pm 29$ & $45 \pm 42$ & $7 \pm 5$ \\
\hline $\begin{array}{l}\text { Relative RBP4 } \\
\text { increase }\end{array}$ & $1,47 \pm 0,35$ & $1,28 \pm 0,41$ & $1,27 \pm 0,49$ & $0,98 \pm 0,39$ & \\
\hline $\begin{array}{l}\text { Relative CRP } \\
\text { increase }\end{array}$ & $13,4 \pm 39,2$ & $10,6 \pm 27,3$ & $21,4 \pm 30,3$ & $57,7 \pm 30,6$ & \\
\hline $\begin{array}{c}\text { Relative SAA } \\
\text { increase }\end{array}$ & $34,5 \pm 104,8$ & $16,0 \pm 45,7$ & $23,7 \pm 47,1$ & $133,9 \pm 61,7$ & \\
\hline
\end{tabular}

Conclusion: This preliminary study showed that RBP4 levels may be increased about 1.5 times in FMF and to lesser extent in AA amyloidosis patients despite no significant change during acute phase response of different infections. Patients with infections show strong CRP and SAA response, and the differential response of RBP4 in FMF patients warrants further analysis in larger group of patients with different clinical characteristics.

Disclosure of Interests: None declared

DOI: 10.1136/annrheumdis-2021-eular.3692

\section{Rehabilitation}

\begin{tabular}{|l|l}
\hline POS1446 & ANTHROPOMETRIC MEASUREMENTS IN UPPER \\
EXTREMITY REHABILITATION OF PATIENTS WITH \\
RHEUMATIC DISEASES
\end{tabular}

P. Herrera-Sandate ${ }^{1}$, D. Vega-Morales ${ }^{1}$, A. L. De-Leon-Ibarra' ${ }^{1}$, P. ValdesTorres $^{2}$, L. A. Chavez-Alvarez ${ }^{1}$, I. D. J. Hernandez-Galarza' ${ }^{1}$, R. Pineda-Sic ${ }^{1}$ D. Á. Galarza-Delgado ${ }^{1}{ }^{1}$ Hospital Universitario Dr. José Eleuterio González, Rheumatology Service, Monterrey, Mexico; ${ }^{2}$ Hospital Universitario Dr. José Eleuterio González, Sports Medicine and Physical Rehabilitation Department, Monterrey, Mexico

Background: The chronic nature of rheumatic diseases has a negative impact in quality of life. Pain and loss of function in the upper extremity cause a progressive difficulty to perform daily activities, often requiring integral physical rehabilitation programs. Anthropometric measurements habitually take prolonged periods of time, given the extensive nature of physical examination in rheumatic patients. It is unknown which factors are most efficient to better reflect the functional status of the rheumatic patient.

Objectives: Determine the most efficient anthropometric measurements in the upper extremity to assess the functional status of rheumatic patients in rehabilitation programs.

Methods: Thirty-six patients were recruited from Rheumatology consultation of University Hospital "Dr. José Eleuterio González" in Monterrey, Mexico. Patients had a complete physical examination by a board-certified rheumatologist, which referred patients to Physical Rehabilitation consultation if necessary. A cross-sectional study was carried out in these patients with Disabilities of the Arm, Shoulder and Hand (DASH) questionnaire (1), measurement of ranges of motion (ROM) and hand strength with the Mathiowetz protocol (2). Analysis was performed through Principal Components Analysis including Total Variance Explained (TVE), Rotated Component Matrix (RCM) and dendrograms.

Results: From the total of patients, $34(94.4 \%)$ were women with a mean age of 34 years (SD 11.33). The most common diagnosis was rheumatoid arthritis $(47.2 \%)$ followed by osteoarthritis $(8.3 \%)$. The mean disability score in DASH was 29.3\% (DE 23.36). The TVE analysis found that 3 elements explained $48.6 \%$ of the total variance, and 13 elements explained $89.4 \%$. RCM correlations among hand strength parameters ranged from 0.51 to 0.93 , whereas ROM correlation values were below 0.4 . Graph 1 depicts data dispersion for ROM, grip strength and pinch gauge by dynamometer.

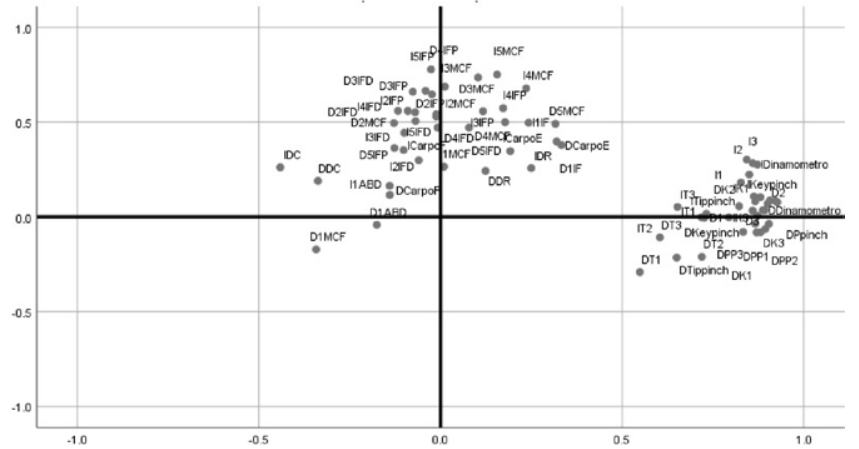

Graph 1. Two main clusters are observed in the RCM plot of the data. In the center and superior to the $x$ axis, a conglomeration corresponding to ROM is shown, consisting of radial and ulnar deviation; flexion, extension, abduction and adduction in carpal bones, metacarpophalangeal joints, and proximal and distal interphalangeal joints of the five fingers of both hands, accordingly. To the right and across the $\mathrm{x}$ axis, another cluster depicts the grip strength and tip, key, and lateral pinch gauge of the fingers according to the Mathiowetz protocol. Closeness of data points portray a higher similarity among variances in the second cluster.

Conclusion: Hand strength is the most efficient parameter to assess the functional status of the upper extremity in rheumatic patients in rehabilitation programs.

\section{REFERENCES:}

[1] Arreguín Reyes, R., López López, C. O., Alvarez Hernández, E., Medrano Ramírez, G., Montes Castillo, M., \& Vázquez-Mellado, J. (2012). Evaluation of hand function in rheumatic disease. Validation and usefulness of the Spanish version AUSCAN, m-SACRAH and Cochin questionnaires. Reumatologia clinica, 8(5), 250-254. https://doi.org/10.1016/j.reuma.2012.03.005

[2] Mathiowetz, V., Kashman, N., Volland, G., Weber, K., Dowe, M., \& Rogers, S. (1985). Grip and pinch strength: normative data for adults. Archives of physical medicine and rehabilitation, 66(2), 69-74.

Disclosure of Interests: None declared

DOI: 10.1136/annrheumdis-2021-eular.1301

\section{POS1447 LOW-INTENSITY PULSED ELECTROMAGNETIC FIELDS IMPROVE PHYSICAL PERFORMANCE IN A DOSE-DEPENDENT MANNER: AN OBSERVATIONAL STUDY IN OLDER ADULTS WITH RHEUMATIC DISEASES}

M. Giovale ${ }^{1}$, G. Tramontano ${ }^{1}$, R. Galli', S. Rando ${ }^{1}$, A. Giusti ${ }^{1}$, L. Bandi ${ }^{1}$, F. Russo ${ }^{1}$, S. Rampoldi ${ }^{2}$, L. C. Bottaro ${ }^{3}$, G. Bianchi ${ }^{1} .{ }^{1}$ Local Health Trust 3, Rheumatology Unit, Department of Medical Specialties, Genoa, Italy; ${ }^{2} \mathrm{THS}$ Therapeutic Solutions, Department of Engineering, Milano, Italy; ${ }^{3}$ Local Health Trust 3, Executive Board, Genoa, Italy

Background: Low-intensity pulsed electromagnetic fields (PEMF) have been shown to improve gait parameters in frail older adults. ${ }^{1}$ Furthermore, the continuous exposure to PEMF (up to 1 year) have been demonstrated to produce progressive improvements in self-selected gait speed in older adults at risk of falling. ${ }^{2}$

Objectives: To investigate the effects of two different treatment regimens of PEMF on physical performances in older adults presenting with rheumatoid arthritis (RA), osteoarthritis (OA) or severe osteoporosis (OP).

Methods: Older adults presenting with RA, OA or OP, at increased risk of falls, evaluated in our Falls Prevention Clinic, were considered for a prospective observational study investigating the effects of PEMF on physical performances. PEMF were supplied by the THS 280 E device (THS-Therapeutic Solutions Srl, Milan, Italy). It provides a new therapeutic approach, named TEPS (Triple Energy Postural Stabilization), that represents an evolution of physical therapy. ${ }^{1,2}$ On the basis of the physician judgment, PEMF were administered following an intensive protocol, every 45 days (PEMF-45), or a standard validated protocol ${ }^{1,2}$, every 60 days (PEMF-60). All subjects were assessed at baseline and every 3 months with the following tests: 4 meters gait speed test [4MGS, seconds (sec)], timed up and go test (TUG, sec), chair stand test (CST, sec), short physical performance battery (SPPB, score), and hand grip strength (HGS) by hand dynamometer $(\mathrm{Kg})$. Demographic, anthropometric and clinical characteristics, including pharmacological treatments and functional status were evaluated at baseline. Clinical and adverse events were assessed every 45 or 60 days after PEMF administration. Results: Overall, 94 patients were enrolled between January and December 2020. Of these, 43 subjects ( $N=33$ PEMF-45, $N=11$ PEMF-60) with a valid 6 -month follow-up assessment were considered for the current analysis. The two groups were comparable regarding the main baseline characteristics, and similar 